\title{
Impaired texture segregation but spared contour integration following damage to right posterior parietal cortex
}

\author{
Kathleen Vancleef · Johan Wagemans • \\ Glyn W. Humphreys
}

Received: 15 January 2013 / Accepted: 18 June 2013

(C) Springer-Verlag Berlin Heidelberg 2013

\begin{abstract}
We examined the relations between texture segregation and contour integration in patients with deficits in spatial attention leading to left or right hemisphere extinction. Patients and control participants were presented with texture and contour stimuli consisting of oriented elements. We induced regularity in the stimuli by manipulating the element orientations resulting in an implicit texture border or explicit contour. Participants had to discriminate curved from straight shapes without making eye movements, while the stimulus presentation time was varied using a QUEST procedure. The results showed that only patients with right hemisphere extinction had a spatial bias, needing a longer presentation time to determine the shape of the border or contour on the contralesional side, especially for borders defined by texture. These results indicate that texture segregation is modulated by attention-related brain areas in the right posterior parietal cortex.
\end{abstract}

Keywords Extinction - Texture segregation - Contour integration $\cdot$ Spatial attention $\cdot$ Posterior parietal cortex . Temporo-parietal junction

\section{Introduction}

Patients showing visual extinction are able to respond to a single item in their contralesional field but not when

K. Vancleef $(\bowtie) \cdot$ J. Wagemans

Laboratory of Experimental Psychology, University of Leuven, Tiensestraat 102, Box 3711, 3000 Leuven, Belgium

e-mail: kathleen.vancleef@psy.kuleuven.be

G. W. Humphreys

Department of Experimental Psychology, University of Oxford, South Parks Road, Oxford OX1 3UD, UK another competing ipsilesional item appears in close temporal proximity. Typically, it is argued that extinction reflects spatial biasing in the competition for attention between two stimuli leading to the inability to attend to the contralesional stimulus in the presence of an ipsilesional competitor (e.g., Duncan et al. 1997). Although extinction is sometimes seen as a mild form of neglect, recent evidence shows it can be distinct phenomena related to distinct lesion locations (Chechlacz et al. 2011; Karnath and Rorden 2012; Riddoch et al. 2010a). Notably, extinction is often associated with lesions in the temporo-parietal junction (TPJ) and middle temporal gyrus (Chechlacz et al. 2011; Karnath and Rorden 2012), while different neglect symptoms are associated with damage to the angular and supra-marginal gyri and to the superior temporal cortex (Chechlacz et al. 2010).

Despite the lack of explicit report, there is evidence that extinguished stimuli are processed in extinction patients. Notably, extinction is reduced when the contralesional stimulus can be grouped with the ipsilesional stimulus, with both stimuli then being selected for report together. Effects of grouping have been noted based on both lowlevel stimulus properties (e.g., collinear edges, common shape and contrast polarity) and higher-level associations (from familiar shape to the lexical identity of words and action relations between pairs of objects) (see Gilchrist et al. 1996; Humphreys 1998; Mattingley et al. 1997; Pavlovskaya et al. 1997; Riddoch et al. 2003; Ward et al. 1994). This evidence suggests that extinguished items can be processed sufficiently to enter into grouping relations with ipsilateral stimuli.

Two candidates for the neural mechanisms underlying perceptual grouping in extinction are long-range horizontal connections in striate and extrastriate cortex and the influence of higher visual areas. First, long-range horizontal 
connections can be responsible for the observed effects of low-level grouping on extinction (see Brooks et al. 2005). For example, there is physiological evidence for the existence of such connections in early visual areas and for their role in basic Gestalt grouping processes (e.g., Gilbert 1993; Malach et al. 1993; Polat and Sagi 1993; for a review, see Wagemans et al. 2012). Interestingly, recovery from extinction between collinear elements reduces with increasing spacing between the elements (Gilchrist et al. 1996), consistent with the role of locally acting horizontal connectivity between neurons in early visual areas (Gilbert 1993; Malach et al. 1993; Polat and Sagi 1993). Pavlovskaya et al. (1997) also observed stronger effects on extinction for two horizontal collinear elements than for two diagonal elements, indicating connectivity constrains in early visual processing (see Polat and Sagi 1994 for converging evidence). A second causal factor can be the influence of higher visual areas. Wilson and Singer (1981) described long-range spatial interactions. They observed interactions between the perception of simultaneously presented flashes in each hemifield with inter-stimulus distances up to 25 degrees, which they attributed to integrative processes in the superior temporal sulcus. In addition, Kumada and Humphreys (2001) showed recovery of extinction when two letters formed a word compared with when they formed a nonword, indicating a role of lexical knowledge. This lexical effect also overcame effects of presenting the letters in opposite contrast polarities, when low-level grouping should be disrupted. Ward et al. (1994) reported a reduction in extinction when elements formed a familiar shape (a horizontal line and a ' $\mathrm{V}$ ' making an arrow) but not with an unfamiliar shape. Riddoch et al. (2003) have reported that extinction is reduced when objects are positioned to interact with one another. Other authors have demonstrated that effects of the task modulate the effects of visual similarity (Baylis et al. 1993). These effects cannot easily be attributed to low-level factors and fit instead with the argument that the co-activation of stored representations by the ipsi- and contralesional items leads to them being selected together.

Although these studies indicate that extinguished items can be processed, the results do not necessarily demonstrate that extinguished items are processed normally. Vuilleumier et al. (2008) used fMRI to measure brain activity to lateralized, high contrast stimuli when a patient showing extinction performed tasks of varying difficulty on fixated stimuli. They found that, as the fixation task became more difficult, there was selectively reduced brain activity on the contralesional side, with the reduction in activity being more pronounced at higher relative to lower visual areas. Han and Humphreys (2007) investigated the coding of proximity, collinearity and similarity in displays comprising several elements grouped in rows or columns in patients with posterior parietal lesions and visual extinction. They showed a reduction in early EEG components to the presence of grouping, consistent with the parietal lesions disrupting grouping.

In the present study, we investigate whether grouping in texture segregation and contour integration, two midlevel visual processes, is still intact in patients with visual extinction after posterior parietal lesions. First, we will present stimuli that group by collinearity to form a single contour (Field et al. 1993). Contour integration is believed to depend mainly on horizontal connections within early visual areas (Hess and Field 1999; Li 1998). For instance, fMRI studies have shown involvement of the striate and extrastriate cortex in collinearity grouping (Altmann et al. 2003) and perception of illusory contours (Seghier and Vuilleumier 2006). In addition, collinearity grouping has been reported to be intact in patients with lesions of higher-level visual regions (Giersch et al. 2000), suggesting that higher-level visual regions may not be necessary for grouping these elements. If this is the case, then collinearity-based grouping should not merely take place, it should be intact in patients with extinction after damage to the posterior parietal cortex. Second, we investigate texture segregation. The neural underpinnings of texture processing are less well understood. There is convincing evidence for early modulation of brain activity in V1 from EEG and single cell studies in man and monkey (Appelbaum et al. 2012; Lamme et al. 1992; Nothdurft et al. 2000; Romani et al. 2003). However, some influence of higher visual areas has also been suggested. El-Shamayleh and Movshon (2011) observed that only a few cells in V2 were responsive to second-order texture segregation and point to V4 as a potential area for texture segregation. Also in an fMRI constrained EEG source-imaging study, texture-based activity proceeds from V1 to V4 and afterward to LOC (Appelbaum et al. 2012). Furthermore, patients with traumatic brain injury showed normal VEP with low levels of visual stimulation, but texture segregation VEP was delayed, suggesting higher-order visual processes were necessary for texture segregation (Lachapelle et al. 2004). In addition, an fMRI study identified V4 and TEO areas as involved in texture segregation (Kastner et al. 2000). Activity from these higher-level visual areas can feed back to striate and extrastriate cortex (Bach and Meigen 1992; El-Shamayleh and Movshon 2011; Romani et al. 2003; Scholte et al. 2008). A possible candidate for the feedback pathway is the TPJ. Piccini et al. (2003) showed that the right TPJ mediated more global processing in early visual areas of a patient with Alzheimer's disease. In addition, functional neuro-imaging studies point to the right TPJ as the source of attentional modulation of activity in early visual areas (Fink et al. 1996). If indeed TPJ is involved in feedback in texture processing, grouping might be impaired in these 
displays as the TPJ is typically damaged in extinction. To test these hypotheses, we presented contour and texture stimuli to patients with visual extinction after posterior parietal lesions and to two control groups (healthy controls and hemianopia patients). The participants had to discriminate curved from straight shaped contours or texture borders. Stimulus presentation time was varied according to an adaptive procedure to estimate the required presentation time to achieve $75 \%$ correct responses. In this way, performance was taken to the same level in all tasks, so that tests of relative effects of grouping in comparing thresholds are not confounded by overall performance differences.

\section{Methods}

\section{Participants}

Between June and October 2011, we tested nine patients with chronic extinction and/or hemispatial neglect after brain damage (age range $=39-78$, mean age $=64.6$ ). Five patients showed left neglect and/or extinction following right hemisphere brain damage (JB, MC, MP, PF, and RP). Four patients had a left hemisphere lesion resulting in right neglect and/or extinction (DT, MH, PH, and RH). For the behavioral experiments on texture segregation and contour integration, two control groups were tested. First, two hemianopic patients participated, one with a left lesion (JE, age $=58)$ and one with a right lesion (DM, age $=64)$ of the primary occipital cortex. These patients were tested for performance in the absence of low-level cortical processing in the contralesional field. Table 1 gives information about the lesions and behavioral deficits of each patient. More details on extinction and neglect measures are presented in Table 2. Second, 12 healthy control participants were tested. These participants were in the same age range as the extinction patients (age range $=45-80$, mean age $=68.7$ ). All participants reported normal or corrected-to-normal visual acuity. MRI scans for the patients were compared with a group of 150 healthy control participants with no neurological deficits spanning the age range of the patients; half of the sample was male, half female.

\section{Behavioral experiments}

\section{Texture segregation and contour integration}

Stimuli The stimuli consisted of Gabor elements positioned on a uniform gray background of approximately 8 by 8 degrees. The Gabor elements were even symmetric and constructed by multiplying a cosine luminance grating with a circular Gaussian. The grating had a spatial frequency of 3.64 cycles per degree, and the Gaussian envelope was characterized by a standard deviation of about 0.1 degrees. These parameter values resulted in Gabor elements of about 0.17 degrees. Gabor elements were placed at quasi-random positions in the display restricted by a minimal inter-element distance of about $0.5^{\circ}$. In the experiment on texture segregation, stimulus displays contained two areas, one at the top and one at the bottom of the stimulus. In each area, all Gabors had the same orientation $\left(0^{\circ}\right.$ or $\left.90^{\circ}\right)$, resulting in two different homogenous regions segregated by local orientation discontinuities at the edge: grouping of elements with the same orientations led to segregation from another grouped area with all elements having an orientation orthogonal to the elements in the first area. In this experiment, the edge between the two regions was not explicitly given, but it ran horizontally from the left to the right side of the display and was centered vertically. In the contour integration experiment, all background elements had a random orientation and the edge was produced by grouping the collinear elements (Fig. 1). The edge defined either by texture segregation or contour integration could take several shapes that can be divided in three conditions: (1) straight, (2) a curved fragment on the ipsilesional side and (3) a curved fragment on the contralesional side. The curved fragments could either curve upward or downward. To balance the occurrence of straight and curved shapes, the straight shape was presented four times as often as each of the curved shapes. The number of Gabor elements was held constant in every display and proportional to the surface area of each region in the texture segregation stimuli. We checked for local density cues with the tools implemented in the GERT toolbox (Demeyer and Machilsen 2011) for MATLAB (Mathworks) that was used to generate the stimuli.

In addition to the Gabor stimuli, we made silhouettes versions and line versions of the stimuli for control experiments. These versions do not contain Gabor elements, but the edge is represented by a maximal contrast difference (Michelson contrast $=0.99$ ): a black and a white region or a black line on a white background (Fig. 1). In this case, the demands on grouping were lessened and therefore we expect extinction patients to be unimpaired. In addition, this control experiment familiarized the participants with the task.

Apparatus and setup Data were collected using two similar setups. All the patients and three healthy control participants (BC, BN, and JD) were tested at the University of Birmingham. In this setup, participants were seated in front of a CRT monitor (View Sonic Professional Series G90fB: 19 inch, screen resolution 1,280 by 1,024 pixels, refresh rate $85 \mathrm{~Hz}$ ) in a dark and silent room. They viewed the center of the stimuli binocularly from a distance of about $75 \mathrm{~cm}$. The other healthy control participants were tested at the University of Leuven. In this setup, stimuli were also presented on 
Table 1 Patient information

\begin{tabular}{|c|c|c|c|c|c|}
\hline Patient & Year of birth & $\begin{array}{l}\text { Cause and year of } \\
\text { brain damage }\end{array}$ & Description of cortical lesion & Behavioral deficits & Papers describing the patient \\
\hline DT & 1941 & Stroke in 2007 & Left lingual and fusiform gyri & $\begin{array}{l}\text { Left hemisphere extinc- } \\
\text { tion }\end{array}$ & No published papers \\
\hline MH & 1953 & Anoxia in 1996 & $\begin{array}{l}\text { Bilateral but more pronounced left } \\
\text { side damage of occipital-parietal } \\
\text { junction, interparietal sulcus and } \\
\text { superior parietal lobe, angular and } \\
\text { supramarginal gyrus, left middle } \\
\text { frontal gyrus }\end{array}$ & $\begin{array}{l}\text { Left hemisphere extinc- } \\
\text { tion, residual chronic } \\
\text { neglect, optic ataxia, } \\
\text { difficulties in activi- } \\
\text { ties of everyday liv- } \\
\text { ing, poor visuo-spatial } \\
\text { memory, dysgraphia, } \\
\text { right-sided muscle } \\
\text { weakness and raised } \\
\text { sensory thresholds }\end{array}$ & $\begin{array}{l}\text { Correani and Humphreys } \\
\text { (2011), Demeyere et al. } \\
\text { (2010), Dombrowe et al. } \\
\text { (2012), Humphreys et al. } \\
\text { (2010), Kitadono and Hum- } \\
\text { phreys (2007), Riddoch } \\
\text { et al. (2004), Snow et al. } \\
\text { (2013), Vivas et al. (2008) }\end{array}$ \\
\hline $\mathrm{PH}$ & 1972 & Stroke in 1999 & $\begin{array}{l}\text { Left inferior frontal cortex extend- } \\
\text { ing posterior to the anterior and } \\
\text { superior temporal lobe }\end{array}$ & $\begin{array}{l}\text { Left hemisphere extinc- } \\
\text { tion, mild hemiple- } \\
\text { gia, limited speech, } \\
\text { agraphia, dyslexia, } \\
\text { aphasia, agrammatism } \\
\text { and some semantic } \\
\text { difficulties }\end{array}$ & $\begin{array}{l}\text { Kumar and Humphreys } \\
\text { (2008) }\end{array}$ \\
\hline RH & 1933 & Stroke in 1999 & $\begin{array}{l}\text { Left inferior parietal cortex: angular } \\
\text { and supramarginal gyrus, superior } \\
\text { and middle temporal lobe, inferior } \\
\text { frontal gyrus }\end{array}$ & $\begin{array}{l}\text { Left hemisphere } \\
\text { extinction, allocentric } \\
\text { neglect in reading and } \\
\text { identifying chimeric } \\
\text { objects, anomia }\end{array}$ & $\begin{array}{l}\text { Correani and Humphreys } \\
\text { (2011), Dombrowe et al. } \\
\text { (2012), Humphreys et al. } \\
\text { (2010), Kitadono and Hum- } \\
\text { phreys (2007, 2009, 2011), } \\
\text { Riddoch et al. (2006), Snow } \\
\text { et al. (2013), Vivas et al. } \\
\text { (2008) }\end{array}$ \\
\hline JB & 1935 & Stroke in 1999 & $\begin{array}{l}\text { Right inferior parietal lobe, angular } \\
\text { gyrus, supramarginal gyrus, } \\
\text { postcentral gyrus, inferior frontal } \\
\text { gyrus; older left occipital lesion }\end{array}$ & $\begin{array}{l}\text { Right hemisphere } \\
\text { extinction and neglect } \\
\text { in reading and writ- } \\
\text { ing, decreased sensa- } \\
\text { tion in left upper limb }\end{array}$ & $\begin{array}{l}\text { Riddoch et al. (2006), Rid- } \\
\text { doch et al. (2010b), Hum- } \\
\text { phreys et al. (2010) }\end{array}$ \\
\hline $\mathrm{MC}$ & 1934 & Stroke in 1998 & $\begin{array}{l}\text { Right occipito-parietal-temporal } \\
\text { areas extending to inferior frontal } \\
\text { gyrus }\end{array}$ & $\begin{array}{l}\text { Right hemisphere } \\
\text { extinction and neglect }\end{array}$ & $\begin{array}{l}\text { Correani and Humphreys } \\
\text { (2011) }\end{array}$ \\
\hline MP & 1947 & Aneurysm in 1992 & $\begin{array}{l}\text { Right supramarginal gyrus and } \\
\text { angular gyrus, superior and middle } \\
\text { temporal gyrus, inferior frontal } \\
\text { gyrus, postcentral gyrus }\end{array}$ & $\begin{array}{l}\text { Right hemisphere extinc- } \\
\text { tion and mild neglect } \\
\text { in scanning tasks, } \\
\text { line bisection, with } \\
\text { chimeric faces and in } \\
\text { reading, dyscalculia }\end{array}$ & $\begin{array}{l}\text { Dombrowe et al. (2012), } \\
\text { Correani and Humphreys } \\
\text { (2011), Humphreys and } \\
\text { Riddoch (2001), Hum- } \\
\text { phreys et al. (2010), Rid- } \\
\text { doch et al. (2010b) }\end{array}$ \\
\hline $\mathrm{PF}$ & 1948 & Stroke in 1995 & $\begin{array}{l}\text { Left superior and inferior parietal } \\
\text { gyrus, left angular gyrus, left supe- } \\
\text { rior temporal gyrus, right inferior } \\
\text { and superior parietal gyrus, right } \\
\text { postcentral gyrus }\end{array}$ & $\begin{array}{l}\text { Right hemisphere } \\
\text { extinction and dys- } \\
\text { graphia }\end{array}$ & $\begin{array}{l}\text { Braet and Humphreys (2007), } \\
\text { Correani and Humphreys } \\
\text { (2011), Dombrowe et al. } \\
\text { (2012), Njomboro et al. } \\
\text { (2008), Vivas et al. (2008) }\end{array}$ \\
\hline RP & 1955 & Stroke in 2005 & $\begin{array}{l}\text { Right inferior parietal gyrus, angular } \\
\text { gyrus, mid occipital lobe, inferior } \\
\text { frontal, mid temporal gyrus, } \\
\text { midfrontal, precentral and } \\
\text { postcentral gyrus }\end{array}$ & $\begin{array}{l}\text { Right hemisphere } \\
\text { extinction and mild } \\
\text { neglect on star } \\
\text { cancelation }\end{array}$ & $\begin{array}{l}\text { Dombrowe et al. (2012), } \\
\text { Humphreys et al. (2010) }\end{array}$ \\
\hline $\mathrm{DM}$ & 1952 & $\begin{array}{l}\text { Evacuation of brain } \\
\text { abscess in } 1994\end{array}$ & $\begin{array}{l}\text { Left medial and inferior occipito- } \\
\text { temporal regions }\end{array}$ & $\begin{array}{l}\text { Left hemisphere } \\
\text { hemianopia, alexia } \\
\text { without agraphia and } \\
\text { anomia, no evidence } \\
\text { for neglect }\end{array}$ & $\begin{array}{l}\text { Gillebert and Humphreys } \\
\text { (2008), Humphreys and } \\
\text { Riddoch (2001) }\end{array}$ \\
\hline $\mathrm{JE}$ & 1948 & Stroke in 2007 & Right lingual gyrus and calcarine & $\begin{array}{l}\text { Right hemisphere } \\
\text { hemianopia }\end{array}$ & No published papers \\
\hline
\end{tabular}


Fig. 1 Examples of stimuli for both experiments. The first two columns show stimuli for the first texture experiment and the control experiment. The last two columns show stimuli for the second contour experiment and the control experiment. The different shape conditions are represented in the different rows

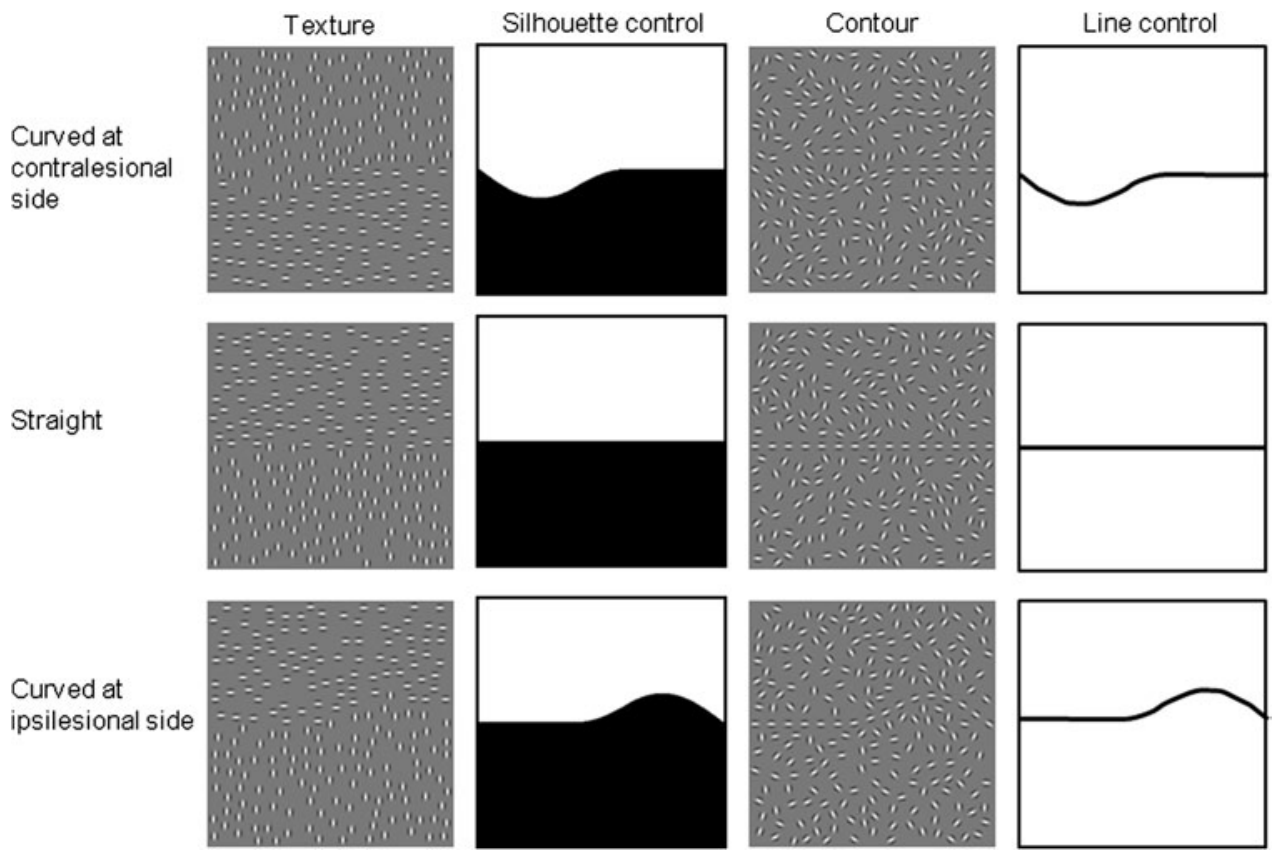

a CRT monitor (Iiyama HM204DT: 21 inch, screen resolution 1,280 by 1,024 pixels, refresh rate $85 \mathrm{~Hz}$ ) and the room was darkened and silent. The stimuli were viewed binocularly from an average distance of $82 \mathrm{~cm}$. The combination of different screen sizes and viewing distances resulted in similar stimulus sizes in both setups. In both setups, eye movements were measured with Eyelink II (SR Research) to monitor fixation during stimulus presentation. The horizontal and vertical gaze positions of one eye were measured at a sampling rate of $500 \mathrm{~Hz}$ in pupil-only mode and were corrected for head movements. The stimuli were presented using Psychtoolbox 3 with MATLAB (MathWorks).

Design and procedure In a Yes/No task, one stimulus was presented on each trial and participants were asked to identify the shape of the curve: is it straight or curved? By comparing performance when a curved fragment fell on the contralesional side with performance when it fell on the ipsilesional side, we measured whether grouping was spatially modulated by the brain lesion. With the exception of two individuals, participants indicated their answer by pressing a key on the keyboard. Because patients DT and MP had difficulties doing this, these patients gave oral responses and the experimenter pressed the corresponding key. To make sure that patients grasped the task, the control experiments in which the difference between curved and straight shapes was readily apparent preceded the corresponding main experiments on texture segregation and contour integration. The order of the main experiments was randomized. Before the start of the experiment, oral and written instructions were given together with at least 10 examples until the participant understood the task. These examples were followed by a practice block of 56 trials. Next, the experiment started and was divided into four blocks of 40 trials. Instructions were repeatedly presented on the screen at the beginning of each block. After 40 trials, the experiment was paused to allow participants to take a break. In the next block, the experiment resumed where the participant left off. Each trial (Fig. 2) started with a fixation cross during which we corrected for drift in eye movement measures. When fixation was stable, a blank screen was presented for $247 \mathrm{~ms}$. Subsequently, the stimulus was presented for a variable time. During stimulus presentation, we controlled for eye movements. This was done because the chronic patients might have learned to make more voluntary eye movements toward their contralesional visual field to compensate for their attentional problem (Hasegawa et al. 2011). Eye movement patterns can be changed during rehabilitation (e.g., Mannan et al. 2010; Vangkilde and Habekost 2010), and Vuilleumier and Schwartz (2001) showed that extinction is influenced by eye movements and eye position. To avoid patients using this compensatory mechanism, we monitored their eye movements. If participants stayed within a window of 1.5 degrees around fixation, the stimulus was followed by a blank screen of $247 \mathrm{~ms}$ and the response cue appeared. If participants made larger eye movements, the trial was aborted and retaken at the end of the block. ${ }^{1}$ To encourage the participants, their response was followed by a feedback tone. We manipulated the presenta-

\footnotetext{
${ }^{1}$ Patient JB and RH found it difficult to stay within a window of $1.5^{\circ}$ around the fixation cross, especially when presented with Gabor stimuli. In order to avoid too many aborted trials and to allow them to complete the experiment, for them no restrictions were made on the initial fixation position.
} 
Fig. 2 Schematic presentation of a trial. The stimulus was one of the examples shown at the right, depending on the experiment

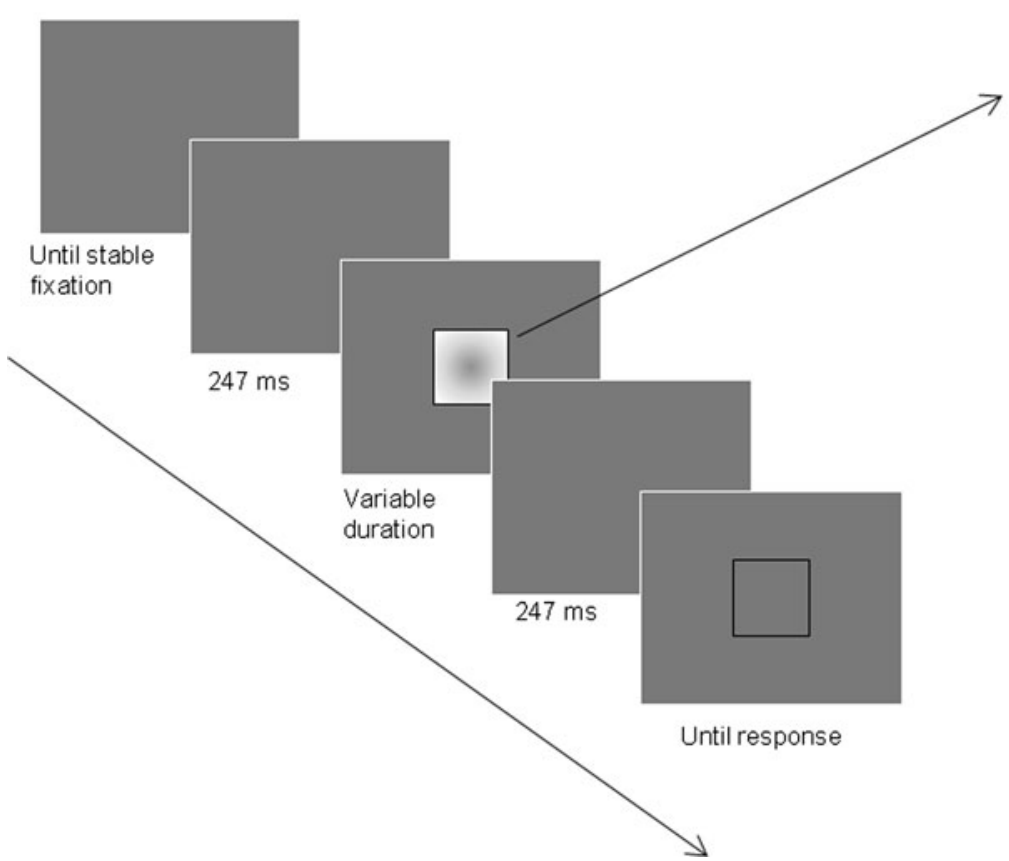

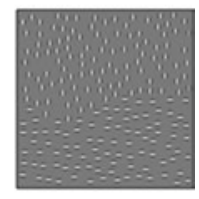
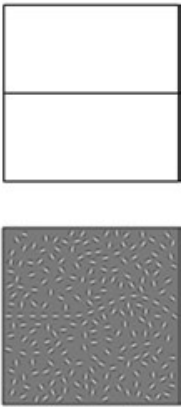

tion time by an adaptive procedure (QUEST) to estimate $75 \%$ correct thresholds. In this procedure, the placement of the trials is guided by the most probable Bayesian estimate of the threshold. The Bayesian estimate is the mode of the posterior probability density function and is based on the data collected thus far and a prior estimate of the threshold. We have set the prior at the mean threshold of the patients during pilot experiments (270 ms for the contours and textures, $12 \mathrm{~ms}$ for the silhouettes and the lines), for more details on the procedure see Watson and Pelli (1983). We estimated the $75 \%$ correct threshold in four intermingled staircases of 40 trials: one staircase for the shape condition with the curve at the ipsilesional side, one for the shape condition with the curve at the contralesional side and two replicating staircases for the straight shape condition because this condition was presented twice as often to balance the occurrence of straight and curved shapes in the experiment. Since threshold performance was measured and taken to the same level across the participant groups, experiments and conditions, overall differences between the groups, experiments and conditions (e.g., differences in cognitive slowing between participant groups or in difficulty level between experiments) are not likely to determine differences in performances; rather our analysis focused on lateralized differences within the conditions with overall task difficulty equated. After each block, feedback about their performance was given to the participants by indicating their percentage correct for this block and the instruction to press the left or right button more often if their proportion left presses ('straight') fell behind or exceeded the proportion of straight shapes by $15 \%$ or more. Participants completed the experi- ment in several sessions and could take a break after each block within a session. The duration of the sessions and the number of blocks within a session depended on the condition of the participant and varied between participants. Healthy control subjects finished the experiment in 2 sessions of 1.5-2 h, and they completed 8-12 blocks within one session. Patients needed 3-5 sessions of $0.5-1.5 \mathrm{~h}$ and could finish 4-9 blocks within a session.

\section{Additional cognitive measurements for patients}

In addition to the measures of grouping, we also examined attention and perception in the patients on several standard measures.

Apple cancellation and visual extinction of $B C o S$ All the patients were tested with the BCoS battery (Humphreys et al. 2012). Two relevant tasks in the context of our study are the Apple Cancellation task and the Visual Extinction task. In the Apple Cancellation task (Bickerton et al. 2011; Chechlacz et al. 2010), object- and space-based neglect can be measured simultaneously. Participants are presented with an A4 sheet in landscape orientation with incomplete and complete outline apples. The incomplete apples had a gap either at their left or right side. The patients were asked to strike through the whole apples. Egocentric neglect was measured by comparing the number of missed targets at the left and right side of the page. Allocentric neglect was measured by comparing the number of left and right false positive responses (cancelations of left and right incomplete apples) across the page. Cutoff scores were set at the 5th 
percentile of the performance of elderly healthy control participants. For egocentric-based neglect, this was three left errors (65 or older) or two left errors (younger than 65) or two right errors (all ages). For allocentric neglect, the cutoff was one left error or one right error for all ages (see Humphreys et al. 2012).

In the Visual Extinction task, the examiner briefly moved a finger in the left visual field (4 trials), in the right visual field (4 trials) or in both fields simultaneously (8 trials), while the patient kept his/her eyes fixed on the examiner's nose. Extinction was measured by comparing the number of correct answers in the right field to the number of correct items in the left field in the bilateral trials. The cutoff for this task was more than 1 error on bilateral trials, for all ages.

Visual extinction at short presentation times Additional finer-grained measures of extinction were also collected (Chechlacz et al. 2011). For this, the patients were presented with the letters A, B, C or D for $200 \mathrm{~ms}$. Either a single letter was presented on the left or at the right side (single-item trials) or two letters were presented, one on the left and one on the right side (bilateral trials) at an eccentricity of $3^{\circ}$. The patients were asked to identify the letters on 48 single-item trials (24 left and 24 right trials) and 48 dual trials while fixating at the center of the screen. Extinction was measured by comparing the number of errors in the right hemifield with the number of errors in the left hemifield on bilateral trials (the asymmetry score). Asymmetry scores greater than two were classified as abnormal, determined by the performance of 29 healthy control participants age-matched to the patients. A second measure was based on a selective drop in performance on bilateral trials compared to single trials (the relative asymmetry score). This was derived by taking twice the difference between left and right hemifield errors on single-item trials and subtracting from that the asymmetry score on bilateral trials. A relative asymmetry score of two was again taken as abnormal based on the healthy control data.

\section{Data analyses behavioral data}

Our main interest was in the relative effects of visual field on texture segregation and contour integration. The field effect was reflected in a difference in the detection threshold for ipsilesional and contralesional curves in the texture segregation and contour integration experiments. We calculated our deficit index in three steps. First, we combined the data from the two identical straight conditions and used these data in a simulated QUEST procedure to obtain a new more stable estimated threshold for the straight conditions based on 80 trials instead of 40 trials. Second, we normalized the threshold for the curved conditions by subtracting the combined estimated threshold of the straight conditions from the estimated thresholds of the curved conditions. Finally, we calculated the difference in normalized estimated thresholds between the two curved conditions. A deficit index close to zero reflects no spatial deficit (no differences between the two curved conditions). A positive deficit index indicates a right spatial deficit (detection of a curve in the right visual field required a higher threshold than detection of a curve in the left field). A negative deficit index indicates a left spatial deficit (detection of the curve in the left field required a higher threshold than detection of a curve in the right field).

For our main statistical analysis, we conducted a $3 \times 4$ repeated measures ANOVA on our deficit measure with group (right extinction, left extinction and healthy control) as a between-subjects factor and experiment (texture, silhouette version, contour, line version) as a within-subjects factor, controlling for eye movements. Eye movements were quantified as the mean Euclidian distance from the fixation cross in each experiment for each participant. To correct for eye movements, we first conducted a linear regression on the deficit measures with eye movements as a predictor. Subsequently, the residuals of this analysis were treated as the dependent variable in a $3 \times 4$ repeated measures ANOVA. The $p$-values for the resulting Type II $F$-statistics of the main effects and the interaction were determined by a nonparametric randomization test. In 5,000 iterations, participants were randomly assigned to the levels of factor group to test the effect of group and the interaction between group and experiment. By randomly assigning the scores of each participant to a level of the factor experiment, we could test the main effect of experiment. On each randomized data set, a $3 \times 4$ repeated measure ANOVA was run. The $F$-statistic observed for the original data set of each main effect and the interaction was compared to the distributions of the corresponding $F$ statistics from the permutations. To determine the $p$-value, we calculated the proportion of permuted $F$-statistics larger or equal to the observed $F$ statistic. Contrasts were calculated following the same principle.

As we had only one left (DM) and one right hemianopic patient (JE), we performed a modified $t$-test (Crawford and Garthwaite 2002; Crawford and Howell 1998) for single cases to compare deficits of the hemianopic patients with the corresponding extinction patients.

Last, we investigated differences between our patient groups for the additional neglect and extinction measures with (modified) $t$-tests. Furthermore, randomization tests for Pearson correlations between our deficit measure and the additional extinction and neglect measures were conducted. In this procedure, we calculated the Pearson correlation between the deficit measure and 
the five additional measures (BCoS Egocentric neglect, BCoS Allocentric neglect, BCoS Extinction, Computerized extinction test Asymmetry score, Computerized extinction test Relative Asymmetry score). Subsequently, we permuted the data as described before and calculated correlations for each permutation. $p$-values for each observed correlation were computed by multiplying the proportion of permuted correlations that were more extreme than the observed correlation by two (two-tailed test). We used Bonferroni method to correct for multiple comparisons.

\section{Brain lesions}

The extinction patients and one hemianopic patient (JE) were scanned on a 3T Philips Achieva MRI scanner at the Birmingham University Imaging Centre. Our second hemianopic patient (DM) had a contra-indication for MRI scanning. A structural scan was acquired with an 8-channel phased array SENSE head coil using a sagittal T1-weigted sequence (sagittal orientation, $\mathrm{TE} / \mathrm{TR}=3.8 / 8.4 \mathrm{~ms}$, voxel size $\left.1 \times 1 \times 1 \mathrm{~mm}^{3}\right)$. After converting and reorienting the images in MRIcron (Chris Rorden, McCausland Center for Brain Imaging, Columbia, SC, USA), the images were preprocessed with Statistical Parametric Mapping (SPM5) software. First, gray matter (GM), white matter (WM) and cerebrospinal fluid (CSF) were segmented in a unified-segmentation procedure and normalized to the Montreal Neurological Institute (MNI) space. Tissue classification was based on an iterative procedure estimating tissue probability maps based on a priori knowledge of expected location of GM, WM and CSF and signal intensity in each voxel. An additional class 'extra' was added to the standard classes GM, WM and CSF that included unexpected and abnormal voxels to improve the classification of damaged tissue (Seghier et al. 2008). Subsequently, normalized and segmented GM and WG images were smoothed with a Gaussian filter of $8 \mathrm{~mm}$ full-with-at-half-maximum (FWHM). In order to define the lesions, the resulting images of each tissue class of the patients were compared with preprocessed images of healthy control participants to detect outlier voxels in each tissue class with an algorithm based on fuzzy clustering (with $\alpha=0.4, \lambda=-4$ and threshold $=0$ ) (for details on the procedure see Seghier et al. 2007, 2008). Subsequently, the resulting outlier maps of GM and WM were fused in a single image giving a continuous lesion definition (fuzzy set) and an outline map. The fuzzy set was thresholded at a voxel intensity of 0.2 and a spatial extent of 100 voxels to generate a binary lesion map (see Seghier et al. 2008). Next, we overlaid binary images of left and of right extinction patients to obtain a spatial distribution map for each patient group.

\section{Results}

Additional cognitive measures

At a group level, there were no significant differences between left hemisphere and right hemisphere patients on $\mathrm{BCoS}$ measures of ego- and allocentric neglect and extinction (egocentric neglect: $t(4)=-1.31, p=0.26$; allocentric neglect: $t(7)=-0.88, p=0.41$; extinction: $t(7)=-2.35, p=0.05)$. In the more stringent computer-based extinction test, there was an overall performance difference $(t(7)=5.48, p=0.001)$. However, we observed no difference in the magnitude of extinction $(t(7)=0.46, p=0.66)$. When comparing hemianopic patients with extinction patients, we found no difference in $\mathrm{BCoS}$ measures of neglect and extinction (JE: egocentric neglect: $t(5)=-0.52, p=0.318$; allocentric neglect: $t(5)=0.40, p=0.359$; extinction: $t(5)=-0.70$, $p=0.266$; DM: egocentric neglect: $t$ statistic could not be calculated because of zero variance; allocentric neglect: $t(4)=-0.79, p=0.238$; extinction: $t(4)=0.72$, $p=0.257)$. In the computerized extinction test, the hemianopic patients were worse at detecting letters in their contralesional relative to their ipsilesional visual field irrespective of the number of stimuli presented (singleitem or bilateral trials), while extinction patients only performed worse on the contralesional side when two letters were presented simultaneously, reflected in a (borderline) significant difference between both groups in the relative asymmetry score (JE: $t(5)=-2.59, p=0.041$; DM: $t(4)=-1.91, p=0.065)$ but not in the absolute asymmetry score (JE: $t(5)=0.86, p=0.227$; DM: $t(4)=0.11, p=0.459)$. Individual scores are presented in Table 2.

\section{Lesion locations}

Overlay images (Fig. 3) of the right hemisphere extinction patients show common lesions in the right supramarginal gyrus and the right inferior parietal lobe. In addition, we found common lesions in the right superior temporal gyrus and the right middle temporal gyrus. Furthermore, the right middle frontal gyrus, the right inferior frontal gyrus and the right postcentral gyrus were damaged in these patients (based on the criterion of damage in at least 4/5 right hemisphere patients and 2/4 left hemisphere patients). The left hemisphere extinction patients showed overlapping lesions in the left middle temporal gyrus. Furthermore, white matter lesions were located in the left superior longitudinal fasciculus and the optic radiation. Patient JE had a right occipital lesion in area 17. An earlier CT scan with DM revealed a lesion in the left inferior medial occipito-temporal region (Humphreys et al. 1997). 
Table 2 Individual scores on additional measures of neglect and extinction

\begin{tabular}{|c|c|c|c|c|c|c|}
\hline \multirow[t]{2}{*}{ Participants } & \multirow[t]{2}{*}{ Deficit $^{\mathrm{a}}$} & \multicolumn{3}{|l|}{$\mathrm{BCoS}$} & \multicolumn{2}{|c|}{ Computerized extinction task } \\
\hline & & $\begin{array}{l}\text { Egocentric } \\
\text { neglect }\end{array}$ & $\begin{array}{l}\text { Allocentric } \\
\text { neglect }\end{array}$ & Extinction & $\begin{array}{l}\text { Asymmetry } \\
\text { score }\end{array}$ & $\begin{array}{l}\text { Relative } \\
\text { asymmetry score }\end{array}$ \\
\hline DT & LE & 0 & 0 & $-8^{*}$ & $18^{*}$ & $14^{*}$ \\
\hline $\mathrm{MH}$ & LE & 0 & -1 & 0 & $7^{*}$ & $7^{*}$ \\
\hline $\mathrm{PH}$ & LE & 0 & 0 & 0 & $35^{*}$ & $25^{*}$ \\
\hline RH & LE & 0 & -1 & $-4 *$ & $30 *$ & $16^{*}$ \\
\hline JB & $\mathrm{RE}$ & -1 & 0 & 1 & $-10^{*}$ & $4 *$ \\
\hline $\mathrm{MC}$ & $\mathrm{RE}$ & $21^{*}$ & 0 & $5^{*}$ & $-30 *$ & $10^{*}$ \\
\hline MP & $\mathrm{RE}$ & $9 *$ & 0 & 0 & $-40 *$ & $20 *$ \\
\hline PF & $\mathrm{RE}$ & 0 & -1 & 0 & $-12 *$ & $10^{*}$ \\
\hline $\mathrm{RP}$ & $\mathrm{RE}$ & -1 & 0 & $8^{*}$ & $-30 *$ & $22 *$ \\
\hline $\mathrm{DM}$ & LH & -1 & -1 & 0 & $24^{*}$ & 0 \\
\hline JE & RH & 0 & 0 & 0 & $-12 *$ & 0 \\
\hline
\end{tabular}

BCoS: negative values represent a right deficit, positive values represent a left deficit; for the asymmetry score on the computer test of extinction: negative values represent a left deficit; positive values represent a right deficit. Scores more extreme than the cutoff values are marked with an *

${ }^{a} L E$ left hemisphere extinction, $R E$ right hemisphere extinction, $L H$ left hemisphere hemianopia, $R H$ right hemisphere hemianopia
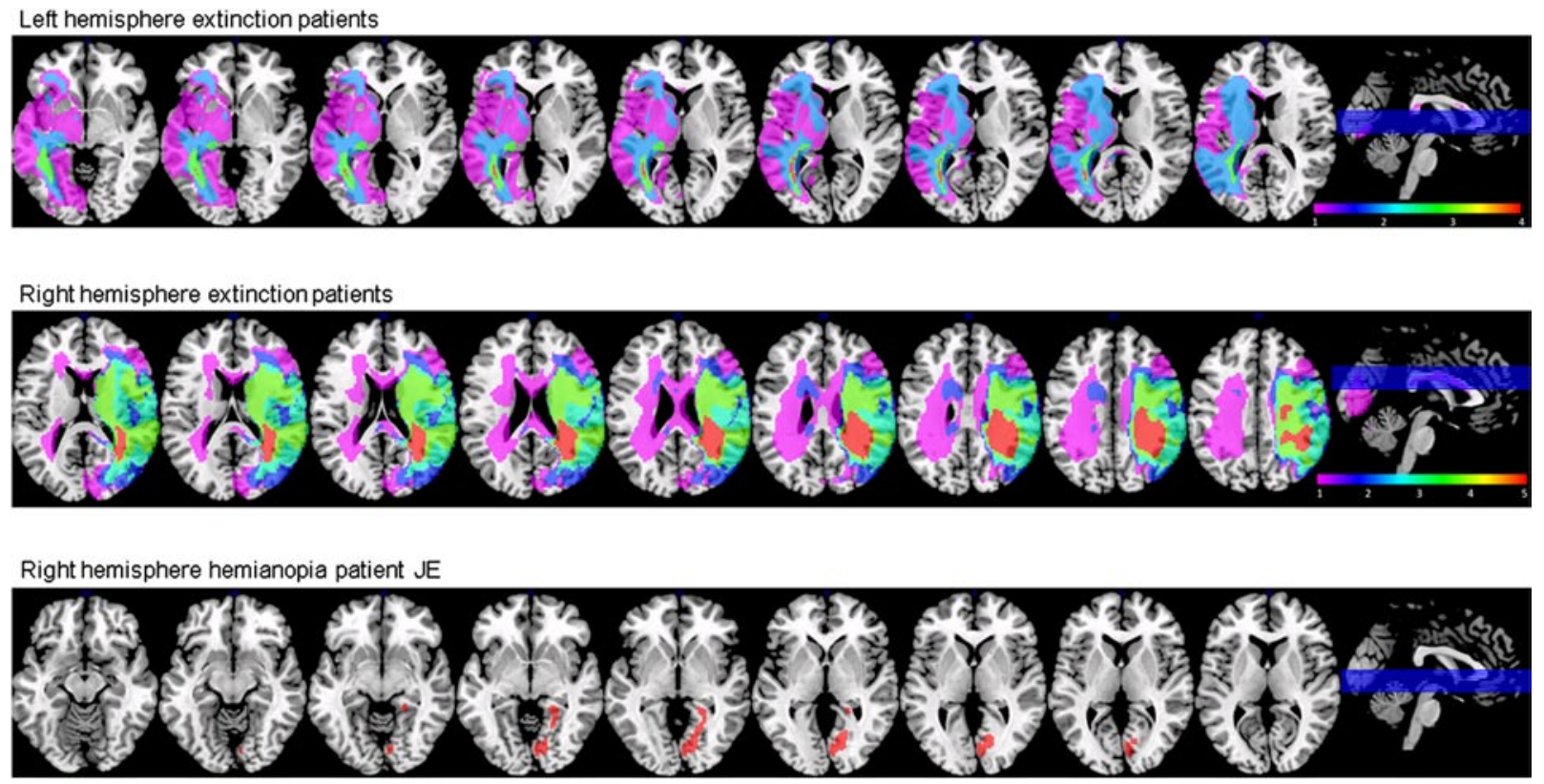

Fig. 3 Sliced overlay images of the patients. The color index indicates the number of patients having a lesion at a certain location. So common lesion locations for all patients in a group are shown in red (four patients in the left hemisphere extinction group, five patients in

Texture segregation and contour integration and correlation with extinction measures

The permutation repeated measures ANOVA (Fig. 4; Table 3) revealed a significant main effect of the group $(F(2,18)=10.01, p=0.003)$, a nonsignificant main effect of experiment (contour vs. texture) $(F(2,30)=0.40$, $p=0.770)$ and a significant interaction between group and the right hemisphere extinction group), while purple indicates only one patient of the group has a lesion at this location (color figure online)

experiment $(F(3,30)=4.31, p=0.001) \cdot{ }^{2,3}$ Contrasts indi-

2 The linear regression analysis showed no significant effect of eye movements on performance when subjects were required to fixate $(t(82)=1.06, p=0.29)$.

3 Analyses were performed with and without including the data from subjects RH and JB, who could freely look at the entire display. Including their data did not change our conclusions. Here, results for all participants are reported. 
Fig. 4 Mean deficit values per group are plotted with an error bar of one standard deviation for each experiment. Negative numbers correspond to a left deficit; positive numbers correspond to a right deficit, while zero represents no deficit (color figure online)

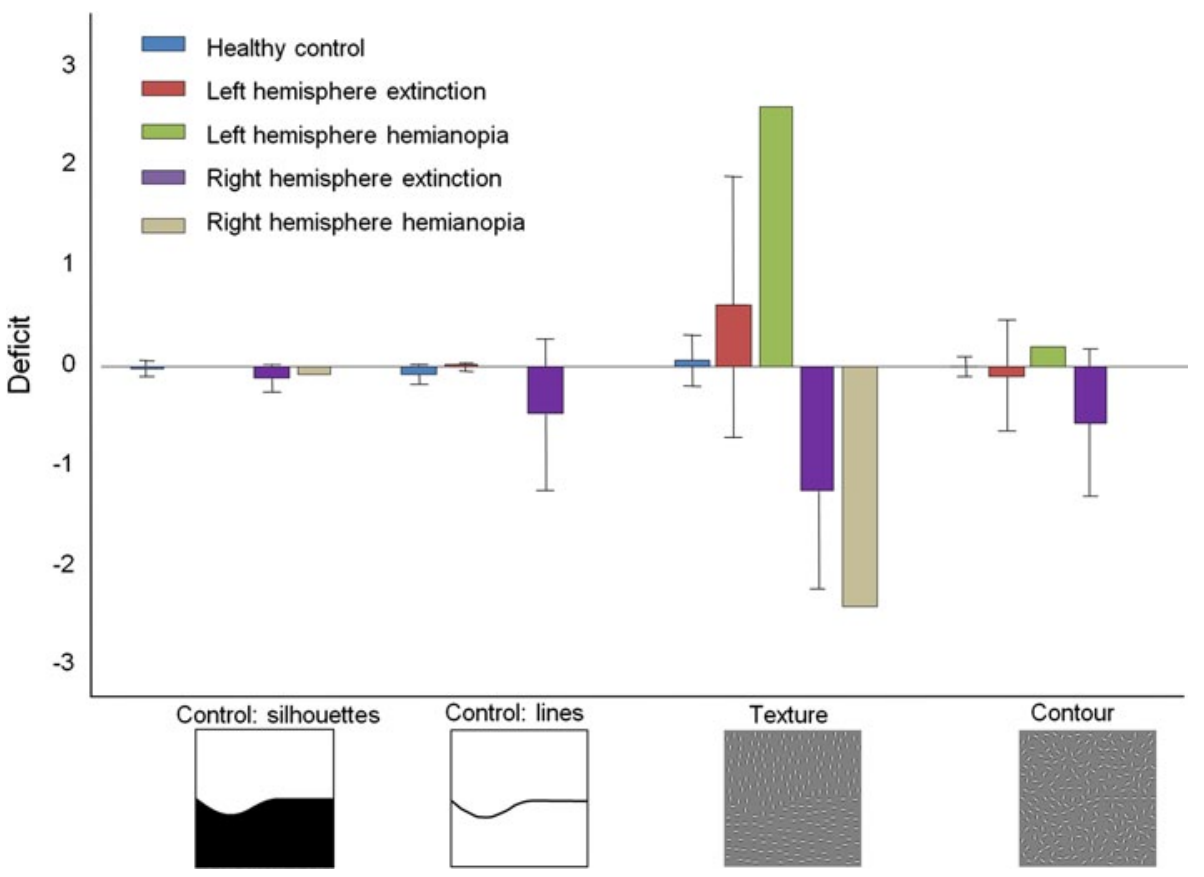

Table 3 Individual deficit measures a $L E$ left hemisphere extinction, $R E$ right hemisphere extinction, $L H$ left hemisphere hemianopia, $R H$ right hemisphere hemianopia, $H C$ healthy control

\begin{tabular}{llcccc}
\hline Participants & Deficit & $\begin{array}{l}\text { Control: silhou- } \\
\text { ettes }\end{array}$ & Control: lines & Texture segregation Contour \\
integration
\end{tabular}

cated that right hemisphere extinction patients performed worse than healthy control participants $(t(72)=-5.45$, $p<0.001$, Bonferroni correction: alpha $=0.006)$ in the texture segregation experiment. In the other experiments, the differences between right hemisphere extinction patients and healthy control participants were not 
Table 4 Statistics for the contrasts between extinction patients and healthy control participants for each experiment

\begin{tabular}{|c|c|c|c|c|}
\hline \multirow[t]{2}{*}{ Experiment } & \multicolumn{2}{|c|}{$\begin{array}{l}\text { Left hemisphere } \\
\text { extinction patients } \\
\text { versus healthy controls }\end{array}$} & \multicolumn{2}{|c|}{$\begin{array}{l}\text { Right hemisphere } \\
\text { extinction patients } \\
\text { versus healthy controls }\end{array}$} \\
\hline & $t^{\mathrm{a}}$ & $p$ & $t^{\mathrm{a}}$ & $p$ \\
\hline $\begin{array}{l}\text { Control: } \\
\text { silhouettes }\end{array}$ & 0.14 & 0.402 & -0.33 & 0.045 \\
\hline Control: lines & 0.3 & 0.880 & -1.74 & 0.016 \\
\hline $\begin{array}{l}\text { Texture } \\
\text { segregation }\end{array}$ & 1.87 & 0.284 & -5.45 & $<0.001^{*}$ \\
\hline $\begin{array}{l}\text { Contour } \\
\text { integration }\end{array}$ & -0.53 & 0.540 & -2.44 & 0.011 \\
\hline
\end{tabular}

significant. There were no significant differences between left hemisphere extinction patients and healthy control participants in any of the experiments (see Table 4). However, the scores on the additional measures of extinction and neglect show that some right hemisphere lesion patients only had extinction (JB, PF and RP), while other right hemisphere lesion patients had both extinction and neglect (MC and MP). Permutation paired $t$-tests were used to compare both groups. These showed that patients with neglect performed significantly worse in the line experiment $(t(72)=3.14, p<0.001)$ and in the contour integration experiment $(t(72)=3.38, p=0.001)$ compared to patients with only extinction. No significant difference was observed between both groups for the silhouettes $(t(72)=0.11, p=0.482)$ and the texture segregation experiment $(t(72)=0.61, p=0.645)$. To compare the hemianopic and extinction patients, we performed a modified $t$-test. The performance of our right hemisphere (left hemianopic) patient, JE, did not differ significantly from the right hemisphere extinction patients in any of the experiments (silhouette control: $t(5)=0.27, p=0.400$; lines control: $t(5)=0.57, p=0.298$; texture: $t(5)=-1.07$, $p=0.172$; contour: $t(5)=0.7, p=0.262$ ). Neither did we find a difference between our left hemisphere hemianopic patient, DM, and the left hemisphere extinction patients (silhouette control: $t(4)=-0.87, p=0.224$; lines control: $t(4)=-0.44, p=0.346$; texture: $t(4)=1.34, p=0.136$; contour: $t(4)=0.47, p=0.336$ ). Compared to healthy control participants, both hemianopic patients showed a significant lower performance in the texture experiment (JE: $t(5)=-9.19, p<0.001$; DM: $t(4)=9.43, p<0.001)$. In the contour experiment, no significant differences were observed (JE: $t(5)=0.04, p=0.486$; DM: $t(4)=1.86$, $p=0.045)$.

Concerning the correlations between performance in our experiments and the additional measures of neglect and extinction, we observed no significant correlation (after Bonferroni correction $\alpha=0.01$ ) between the deficit index in our main experiments with the ego- and allocentric neglect measures in the $\mathrm{BCoS}$ Apple Cancellation task (Egocentric neglect measure: $r=-0.40 p=0.15$, Allocentric neglect measure: $r=-0.12 p=0.72$ ) and nor was there a strong correlation with the visual extinction test $(r=-0.38, p=0.12)$ of the BCoS. For the stringent computer-based extinction task, we found a significant correlation between our deficit measure and the asymmetry score $(r=0.61, p<0.001)$ in the extinction patients. When comparing the magnitude of the deficit (absolute values of our original measure) with the relative asymmetry score, we found no significant correlation $(r=0.28, p=0.20)$.

\section{Discussion}

Our major results indicate that texture segregation is impaired in right hemisphere extinction patients, while contour integration is intact in left and right hemisphere extinction patients. The results suggest a specific role for higherorder brain regions in the right hemisphere (see below) in texture segregation. However, an alternative proposal is that the data reflect different filtering processes instead of a fundamental contrast between contour integration and texture segregation. While shape discrimination of borderline in the texture segregation stimuli requires second-order filtering processes, the contours in our contour integration stimuli can be picked up by a first-order filter. Especially when all contour elements are aligned, as in the straight contour condition, the resultant contour can be picked up by a large scale first-order orientation filter and no linking of elements by a second-order filter is necessary. Hess and Dakin (1997) compared first-order filtering predictions and human performance in contours with constant phases and with alternating phases of the Gabor elements. Since alternating phase contours require additional linking, firstorder models predict that performance should be at chance with this type of contour. However, Hess and Dakin (1997) found that human performance did not follow these predictions of a first-order filter model with centrally presented contours of high curvature: contours with alternating phase Gabors only induced a small decrease in performance compared to contours with constant phase Gabors. This is consistent with linkage taking place between orientation selective cells. In contrast, with presentations beyond $10^{\circ}$ eccentricity, performance dropped dramatically and there was no evidence for linkage taking place. Since our stimuli are only $8^{\circ}$ in size, we expect the stimuli will involve element linking. However, we cannot exclude the first-order filtering hypothesis because-even though it might be inefficient for the visual system to use a different mechanism 
for straight contours than for curved contours-the prediction of a filtering-only model for straight constant phase contours is similar to human performance. Also since the subjects of Hess and Dakin (1997) performed a contour detection task, while our subjects perform a shape discrimination task, the processing mechanism might be different depending on the task.

\section{Control experiment: contours with alternating phase Gabors}

\section{Introduction}

To check if our straight contour stimuli were processed by a first-order filter, we designed a control experiment in which participants performed the same shape discrimination task on contours with constant phase Gabors and on contours with alternating phase Gabors. A large decline in performance in the alternating phase contours would be indicative of a first-order filtering process only, without element linkage. In addition, we would expect to see this effect only with straight contours since, in the curved contour condition, the linking of elements is necessary to account for the curvature (Hess and Dakin 1997). To familiarize the subjects with the task, the lines experiment was repeated at the beginning.

\section{Methods}

In these control experiments, five healthy control participants matched in age to our neuropsychological patients (age range $=73-80$, mean age $=76$ ) were tested. All participants reported normal or corrected-to-normal vision. The stimuli were similar to the previously described line and contour stimuli. In addition to the original line stimuli and the contour stimuli with constant phase of the Gabors elements, we also included contour stimuli with alternating phase of the Gabor contour elements between $0^{\circ}$ and $180^{\circ}$. The phase of the Gabor elements in the background was randomly set to $0^{\circ}$ or $180^{\circ}$. The participants were tested at the University of Leuven. Stimuli were presented on a calibrated CRT monitor (the same type as described above) at a distance of $82 \mathrm{~cm}$, resulting in stimuli of 8 by 8 degrees. Participants had to identify the shape of the curve in three experiments: lines, contour-same phase and contour-alternating phase. The experiments followed the procedures that were described above.

\section{Results and discussion}

As can be seen in Fig. 5, in all shape conditions threshold estimates were $8-12 \mathrm{~ms}$ higher for contours with

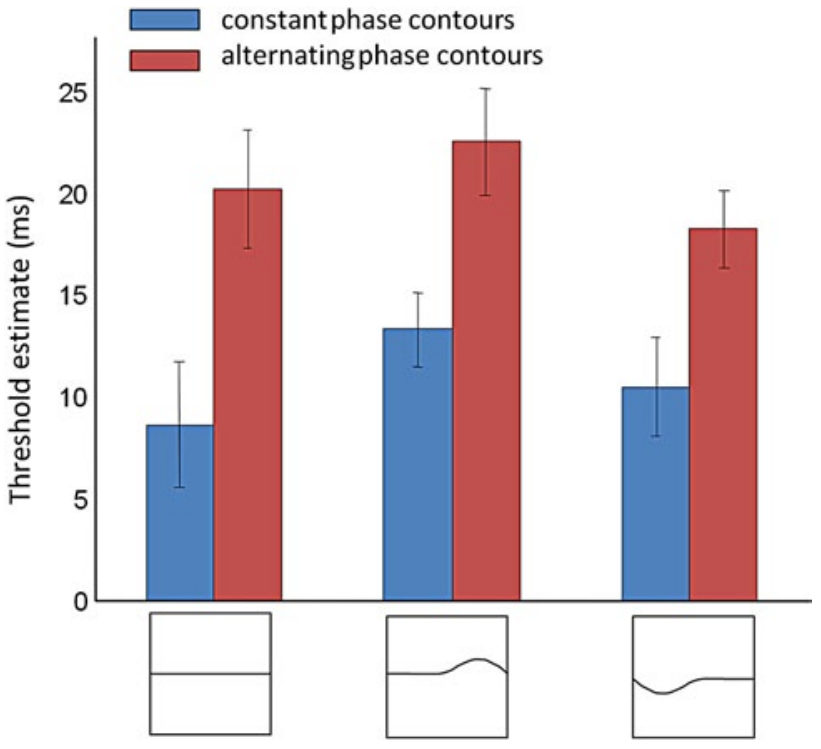

Fig. 5 Estimated threshold average over subjects are plotted for contours with constant phase Gabors (blue) and contours with alternating phase Gabors (red). The error bars indicate 1.96 standard deviations below and above the mean threshold estimates. The icons below the graph illustrate the shape conditions and not the actual stimuli that were presented in the experiment (color figure online)

alternating phase Gabors compared to contours with constant phase Gabors. This was confirmed by a repeated measures ANOVA which indicated a significant effect of phase $(F(1,4)=11.67, p=0.027)$ and a nonsignificant interaction between phase and shape $(F(2,8)=0.6027$, $p=0.570$ ). Given that our subjects did not perform at chance level for the contours with alternating phase Gabors (and only slightly slower than when phases were aligned), we argue that a first-order filter process without element linking is unlikely to underlie the processing of our stimuli. In addition, the effect of phase was the same for the straight contours as for the curved contours in which linking is necessary to perceive the curvature. We conclude that performance in our contour stimuli was most probably based on contour integration by linking of elements and not by simple cortical filtering. Note that contour linkages also seem likely for our extinction patients since they could perform above chance when contour integration was demanded on curved stimuli.

\section{General discussion}

We found a clear spatial deficit for right hemisphere extinction patients in texture segregation. In the contour integration and in the line control experiment, we found no spatial deficit for left hemisphere extinction patients and a small and nonsignificant effect for right hemisphere extinction 
patients. This indicates right hemisphere extinction patients have impaired grouping processes in texture segregation. Left hemisphere extinction patients performed equally well as healthy control participants in all tasks, indicating grouping is still intact in these patients. In addition, we found no significant differences in performance between the extinction patients and the hemianopic patients, suggesting that the functional deficit in the extinction patients was equivalent to that of pure sensory loss. For the extinction patients, performance in the main experiment was correlated with performance in the computerized extinction task in which stimuli are presented at shorter presentation times than in classical neglect and extinction measures, with which we found no correlation.

Our findings are in accordance with the literature on the role of feedback from higher visual areas in texture processing. It has been shown that primary texture processing takes place in V1 (Appelbaum et al. 2012; Lamme et al. 1992; Nothdurft et al. 2000; Romani et al. 2003), but can be modulated by feedback from higher visual areas (Romani et al. 2003; Scholte et al. 2008) like V4, TEO and LOC (Appelbaum et al. 2012; El-Shamayleh and Movshon 2011; Kastner et al. 2000; Lachapelle et al. 2004). Other studies suggest that this feedback runs through the TPJ (Fink et al. 1996; Piccini et al. 2003). Extinction is associated with damage to the TPJ (Chechlacz et al. 2011; Karnath and Rorden 2012), and regions around this area were damaged in our patients (Fig. 3). Hence, we expected this feedback loop to be damaged resulting in impaired processing of textures and that is indeed what we found. However, one should keep in mind that the TPJ is not clearly defined (Gillebert et al. 2013). A lesion-symptom mapping study including more patients would be advisable to draw solid conclusions on the role of specific areas in the parietal cortex in texture processing. Nevertheless, together with previous findings, our results suggest that texture segregation requires attentional control that is probably mediated by the right TPJ for intact grouping of texture elements. Horizontal connections in the occipital cortex appear not sufficient to group the elements in texture displays.

In classical models of texture segregation, such as filterrectify-filter models (reviewed in Landy and Graham 2004) and region-based models (Caelli 1985; Jain and Farrokhnia 1991), filtering and element linking are attributed to early visual areas and texture segregation is thought to be a pre-attentive process (Nothdurft 1992). In these models, modulation of texture processing by spatial attention is not included. However, later models that integrate the principles of classical models include the possibility of top-down influences of higher visual areas on texture segregation. For instance, Roelfsema et al. (2002) designed a model of feed-forward and feedback loops between areas V1, V2 and $\mathrm{V} 3$ and temporal regions TEO and TE of the monkey brain.
Bhatt et al. (2007) go a step further and explicitly model a role for a spatial attention 'shroud' in texture segregation. They attributed their spatial attention component to an interaction between boundary-gated filling-in and longrange inter-surface competition in the posterior parietal cortex. Such models of texture modulation by spatial attention are supported by our findings that texture segregation is disturbed by spatial attention deficits.

In addition, the lateralization of our effect is in accordance with the previous literature: We found intact grouping in patients with a left hemisphere lesion but not in patients with a right hemisphere lesion. Similarly, the majority of previous studies on neglect and extinction have reported more severe behavioral deficits after right hemisphere brain damage than after left hemisphere brain damage (Chechlacz et al. 2010; Chechlacz et al. 2011; Karnath and Rorden 2012; Riddoch et al. 2010a). Furthermore, our result is in agreement with previous studies indicating the importance of the right TPJ (Piccini et al. 2003) for global processing.

In addition to the influence of high-level visual areas in texture segregation, we also found support for the role of horizontal connections in contour integration grouping (Brooks et al. 2005; Gilchrist et al. 1996; Pavlovskaya et al. 1997, 2000; Vuilleumier and Rafal 2000). We extended the findings of previous studies on collinearity by presenting more than two or three elements (cf. Gilchrist et al. 1996; Humphreys 1998; Humphreys et al. 1994; Mattingley et al. 1997; Pavlovskaya et al. 1997, 2000; Ward et al. 1994). Similarly to these studies, we observed intact grouping and therefore recovery from extinction for collinearity grouping in both left and right hemisphere extinction patients. A control experiment with contours with constant and alternating phases of the Gabor elements indicated that first-order filtering only is unlikely to have given rise to our results, but that linking between elements is necessary to perform the task (at least in healthy control subjects). Several authors suggested that horizontal connections in V1 contribute to collinearity grouping in contour integration (Field et al. 1993; Li 1998). In a review of the literature, Loffler (2008) concluded that horizontal connections are likely involved in linking contour elements. However, for shape perception, as was required in our experiment, these local interactions are not sufficient and he suggested the need for a contribution from higher visual areas and possibly feedback to earlier visual regions. Our results do not exclude top-down influences from, for instance, intact shape-processing areas LOC, LO and frontal areas (Aglioti et al. 1997; Baylis et al. 1993; Ptak et al. 2002) and can therefore be interpreted in accordance with this view. However, two patients with a right hemisphere lesion did show impaired performance in the contour integration task. These patients also showed neglect on our additional measures and performed worse 
on the line control experiment. This suggests that contour grouping might be impaired after damage to the angular and supra-marginal gyri and to the superior temporal cortex-areas associated with neglect and not with extinction (Chechlacz et al. 2010). However, to make firm conclusions about the relation between contour integration and associated brain areas more patients with either neglect, extinction or both should be compared. In sum, we suggest that the presence of horizontal connections in early cortical regions in extinction patients, along with support from shape-processing regions, enables contour integration to take place and these processes are not disrupted by damage to the TPJ.

Most models of contour integration do not include a spatial attention component. For instance in the association field model (Field et al. 1993), the biological substrates of the association fields are limited to long-range connections in V1. Also in the models of Li (1998), Ursino and La Cara (2004), VanRullen et al. (2001) and Yen and Finkel (1998), there is no need to include higher visual areas or feedback. Our results support the idea that local processing in V1 is enough to emphasize contours, and there is then no need for high-level parietal structures-though, involvement of shape perception areas (V2 and V4), including feedback from these areas that is postulated by other contour integration models (Hess and Field 1999; Li et al. 2008; Ross et al. 2000), cannot be eliminated.

Differences in difficulty levels between texture segregation, contour integration and the control experiments might be raised as an alternative explanation for the observed effects. However, we took several measures to avoid the role of task difficulty. First, we manipulated presentation times using adaptive procedure to set performance at $75 \%$ correct in each task. In this way, performance levels were similar in all experiments. Second, we normalized our threshold estimate for the curved conditions by subtracting the baseline for each participant and for each experiment. Therefore, additional differences in overall performance levels between participants and between experiments were removed. Last, our relevant dependent measure was a relative difference in performance between two conditions within one experiment: we compared the normalized threshold estimates of the curved conditions within each experiment. We believe that with these procedures, we minimized the role played by differences in task difficulty.

We observed similar effects for the extinction patients as for the hemianopic patients, although only one extinction patient (MC) had damage encroaching on primary visual cortex. This suggests that although the extinction patients do not have a visual field deficit, they perform similarly to the hemianopic patients who do have sensory loss in their contralesional field. Plausibly, the occipital cortex provides the source of the signals entering into contour and texture processing, while the parietal cortex mediates feedback to the occipital areas. The good performance of the hemianopic patients in the control conditions and also with contour integration stimuli may indicate that the field loss was not absolute, but enabled, for instance, local contour interactions to support performance in the control and contour conditions, while being insufficient to support feedback from higher visual regions in the texture segmentation task. So, similar performance between the extinction patients and the hemianopic patients indicates that the affected visual field is functionally blind to texture differences. The right hemianopic patient, DM, did not differ statistically from controls in any task, though, as for the other hemianopic patient JE there was a clear trend for impaired performance but only in the texture condition (Fig. 4).

The high correlation between performance in our experiments and performance in the computerized extinction task is consistent with similar constraints operating on both tasks and with these tasks being more sensitive to attentional limitations than more clinical tests (as in the BCoS) (see also Kaplan et al. 1995; Riddoch et al. 2010a).

Our finding that contour integration is still intact in extinction patients might provide an important cue for patient rehabilitation. Visual spatial attention can be aided by strong contour grouping and induce recovery from extinction. Combining texture surface and contour information improves shape detection as was shown by Machilsen and Wagemans (2011) and can therefore help patients to direct attention to their contralesional visual field and perceive stimuli on this side that were previously outside their awareness.

In sum, our main results indicate that texture segregation is modulated by attention-related brain areas in the right hemisphere, such as the right temporo-parietal junction (TPJ), which is typically damaged in extinction. For contour integration, horizontal connections in the occipital cortex can be sufficient for efficient grouping, although shape processing of the contour can be mediated by high-level visual areas in the temporal cortex.

Acknowledgments This work was supported by the Methusalem program by the Flemish Government (METH/08/02) awarded to Johan Wagemans and by travel grants of Academische Stichting Leuven and Fund for Scientific Research (FWO-Vlaanderen) awarded to Kathleen Vancleef. In addition, support was provided by the Stroke Association and the National Institute of Health Research (UK). We would like to thank Karen Lefever and Lien Peters for assistance in data collection, the patients for their kind participation and two reviewers for their helpful comments.

\section{References}

Aglioti S, Smania N, Barbieri C, Corbetta M (1997) Influence of stimulus salience and attentional demands on visual search patterns in 
hemispatial neglect. Brain Cogn 34(3):388-403. doi:10.1006/b rcg.1997.0915

Altmann CF, Bülthoff HH, Kourtzi Z (2003) Perceptual organization of local elements into global shapes in the human visual cortex. Curr Biol 13:342-349. doi:10.1016/S0960-9822(03)00052-6

Appelbaum LG, Ales JM, Norcia AM (2012) The time course of segmentation and cue-selectivity in the human visual cortex. PLoS ONE 7(3):e34205. doi:10.1371/journal.pone.0034205

Bach M, Meigen T (1992) Electrophysiological correlates of texture segregation in the human visual evoked potential. Vision Res 32(3):417-424. doi:10.1016/0042-6989(92)90233-9

Baylis GC, Driver J, Rafal RD (1993) Visual extinction and stimulus repetition. J Cogn Neurosci 5(4):453-466. doi:10.1162/j ocn.1993.5.4.453

Bhatt R, Carpenter GA, Grossberg S (2007) Texture segregation by visual cortex: perceptual grouping, attention, and learning. Vision Res 47(25):3173-3211. doi:10.1016/j.visres.2007.07.013

Bickerton W-L, Samson D, Williamson J, Humphreys GW (2011) Separating forms of neglect using the Apples test: validation and functional prediction in chronic and acute stroke. Neuropsychology 25(5):567-580. doi:10.1037/a0023501

Braet W, Humphreys G (2007) A selective effect of parietal damage on letter identification in mixed case words. Neuropsychologia 45(10):2226-2233. doi:10.1016/j.neuropsychologia.2007.02.016

Brooks JL, Wong Y, Robertson LC (2005) Crossing the midline: reducing attentional deficits via interhemispheric interactions. Neuropsychologia 43:572-582. doi:10.1016/j.neuropsycholo gia.2004.07.009

Caelli T (1985) Three processing characteristics in visual texture segmentation. Spat Vis 1(1):19-30

Chechlacz M, Rotshtein P, Bickerton W-L, Hansen PC, Deb S, Humphreys GW (2010) Separating neural correlates of allocentric and egocentric neglect: distinct cortical sites and common white matter disconnections. Cogn Neuropsychol 27(3):277-303. doi:10.10 80/02643294.2010.519699

Chechlacz M, Rotshtein P, Hansen PC, Deb S, Riddoch MJ, Humphreys GW (2011) The central role of the temporo-parietal junction and the superior longitudinal fasciculus in supporting multi-item competition: evidence from lesion-symptom mapping of extinction. Cortex (in press). doi:10.1016/j.cortex. 2011.11.008

Correani A, Humphreys GW (2011) An impaired attentional dwell time after parietal and frontal lesions related to impaired selective attention not unilateral neglect. Cogn Neuropsychol 28(5):363385. doi: $10.1080 / 02643294.2012 .656587$

Crawford JR, Garthwaite PH (2002) Investigation of the single case in neuropsychology: confidence limits on the abnormality of test scores and test score differences. Neuropsychologia 40(8):11961208. doi:10.1016/S0028-3932(01)00224-X

Crawford JR, Howell DC (1998) Comparing an individual's test score against norms derived from small samples. Clin Neuropsychol 12(4):482-486. doi:10.1076/clin.12.4.482.7241

Demeyer M, Machilsen B (2011) The construction of perceptual grouping displays using GERT. Behav Res Method 44(2):439446. doi:10.3758/s13428-011-0167-8

Demeyere N, Lestou V, Humphreys GW (2010) Neuropsychological evidence for a dissociation in counting and subitizing. Neurocase 16(3):219-237. doi:10.1080/13554790903405719

Dombrowe I, Donk M, Wright H, Olivers CNL, Humphreys GW (2012) The contribution of stimulus-driven and goal-driven mechanisms to feature-based selection in patients with spatial attention deficits. Cogn Neuropsychol 29(3):249-274. doi:10.10 80/02643294.2012.712509

Duncan J, Humphreys GW, Ward R (1997) Competitive brain activity in visual attention. Curr Opin Neurobiol 7(2):255-261. doi:10.1016/S0959-4388(97)80014-1
El-Shamayleh Y, Movshon JA (2011) Neuronal responses to texturedefined form in macaque visual area V2. J Neurosci 31(23):85438555. doi:10.1523/JNEUROSCI.5974-10.2011

Field DJ, Hayes A, Hess RF (1993) Contour integration by the human visual system: evidence for a local "association field". Vision Res 33(2):173-193. doi:10.1016/0042-6989(93)90156-Q

Fink GR, Halligan P, Marshall J, Frith CD, Frackowiak R, Dolan RJ (1996) Where in the brain does visual attention select the forest and the trees? Nature 382:626-628. doi:10.1038/382626a0

Giersch A, Humphreys GW, Boucart M, Kovacs I (2000) The computation of occluded contours in visual agnosia: evidence for early computation prior to shape binding and figure-ground coding. Cogn Neuropsychol 17(8):731-759. doi: $10.1080 / 026432900750038317$

Gilbert CD (1993) Circuitry, architecture and functional dynamics of visual cortex. Cereb Cortex 3:373-386. doi:10.1093/cer cor/3.5.373

Gilchrist ID, Humphreys GW, Riddoch MJ (1996) Grouping and extinction: evidence for low-level modulation of visual selection. Cogn Neuropsychol 13(8):1223-1249. doi: $10.1080 / 026432996381737$

Gillebert CR, Humphreys GW (2008) Neuropsychological evidence for a spatial bias in visual short-term memory after left posterior ventral damage. Cogn Neuropsychol 25(3):319-342. doi:10.1080/02643290801940558

Gillebert CR, Mantini D, Peeters R, Dupont R, Vandenbergh R (2013) Cytoarchitectonic mapping of attentional selection and reorienting in parietal cortex. NeuroImage 67:257-272. doi:10.1016/j.ne uroimage.2012.11.026

Han S, Humphreys GW (2007) The fronto-parietal network and topdown modulation of perceptual grouping. Neurocase 13(4):278289. doi:10.1080/13554790701649930

Hasegawa C, Hirono N, Yamadori A (2011) Discrepancy in unilateral spatial neglect between daily living and neuropsychological test situations : a single case study. Neurocase 17(6):518-526. doi:10. 1080/13554794.2010.547506

Hess RF, Dakin SC (1997) Absence of contour linking in peripheral vision. Nature 390(6660):602-604. doi:10.1038/37593

Hess RF, Field DJ (1999) Integration of contours: new insights. Trend Cogn Sci 3(12):480-486

Humphreys GW (1998) Neural representation of objects in space: a dual coding account. Philos Trans R Soc B Biol Sci 353:13411351. doi:10.1016/S1364-6613(99)01410-2

Humphreys GW, Riddoch MJ (2001) Knowing what you need but not what you want: affordances and action-defined templates in neglect. Behav Neurol 13(1-2):75-87

Humphreys GW, Romani C, Olson A, Riddoch MJ, Duncan J (1994) Non-spatial extinction following lesions of the parietal lobe in humans. Nature 372:357-359. doi:10.1038/372357a0

Humphreys GW, Riddoch MJ, Price C (1997) Top-down processes in object identification: evidence from experimental psychology, neuropsychology and functional anatomy. Philos Trans R Soc B Biol Sci 352:1275-1282

Humphreys GW, Wulff M, Yoon EY, Riddoch MJ (2010) Neuropsychological evidence for visual- and motor-based affordance: effects of reference frame and object-hand congruence. J Exp Psychol Learn Mem Cogn 36(3):659-670. doi:10.1037/a0019317

Humphreys GW, Bickerton W-L, Samson D, Riddoch MJ (2012) $\mathrm{BCoS}$ cognitive screen. Psychology Press, London

Jain AK, Farrokhnia F (1991) Unsupervised texture segmentation using Gabor filters. Pattern Recogn 24(12):1167-1186. doi:10.1016/0031-3203(91)90143-S

Kaplan RF, Cohen RA, Rosengart A, Elsner AE, Hedges TR, Caplan LR (1995) Extinction during time controlled direct retinal stimulation after recovery from right hemispheric stroke. J Neurol Neurosurg Psychiatry 59(5):534-536. doi:10.1136/jnnp.59.5.534 
Karnath H-O, Rorden C (2012) The anatomy of spatial neglect. Neuropsychologia 50(6):1010-1017. doi:10.1016/j.neuropsycholo gia.2011.06.027

Kastner S, Weerd PD, Ungerleider LG, Lulé D, Diekmann V, Müller $\mathrm{H}$, Kassubek J et al (2000) Texture segregation in the human visual cortex: a functional MRI study. J Neurophysiol 83:2453-2457

Kitadono K, Humphreys GW (2007) Interactions between perception and action programming: evidence from visual extinction and optic ataxia. Cogn Neuropsychol 24(7):731-754. doi:10.1080/02643290701734721

Kitadono K, Humphreys GW (2009) Sustained interactions between perception and action in visual extinction and neglect: evidence from sequential pointing. Neuropsychologia 47(6):1592-1599. doi:10.1016/j.neuropsychologia.2008.11.010

Kitadono K, Humphreys GW (2011) Neuropsychological evidence for an interaction between endogenous visual and motor-based attention. Neurocase 17(4):323-331. doi:10.1080/13554794.2010.509322

Kumada T, Humphreys GW (2001) Lexical recovery from extinction: interactions between visual form and stored knowledge modulate visual selection. Cogn Neuropsychol 18(5):465-478. doi:10.1080/02643290042000224

Kumar VP, Humphreys GW (2008) The role of semantic knowledge in relearning spellings: evidence from deep dysgraphia. Aphasiology 22(5):489-504. doi:10.1080/02687030701517206

Lachapelle J, Ouimet C, Bach M, Ptito A, McKerral M (2004) Texture segregation in traumatic brain injury-a VEP study. Vision Res 44(24):2835-2842. doi:10.1016/j.visres.2004.06.007

Lamme VAF, Van Dijk BW, Spekreijse H (1992) Texture segregation is processed by primary visual cortex in man and monkey. Evidence from VEP experiments. Vision Res 32(5):797-807. doi:10.1016/0042-6989(92)90022-B

Landy MS, Graham N (2004) Visual perception of texture. In: Chalupa LM, Werner JS (eds) The visual neurosciences, vol 169. MIT Press, Cambridge, MA, pp 1106-1118

Li Z (1998) A neural model of contour integration in the primary visual cortex. Neural Comput 10:903-940. doi:10.1162/ 089976698300017557

Li W, Piëch V, Gilbert CD (2008) Learning to link visual contours. Neuron 57(3):442-451. doi:10.1016/j.neuron.2007.12.011

Loffler G (2008) Perception of contours and shapes: low and intermediate stage mechanisms. Vision Res 48(20):2106-2127. doi:10.1016/j.visres.2008.03.006

Machilsen B, Wagemans J (2011) Integration of contour and surface information in shape detection. Vision Res 51(1):179-186. doi:10.1016/j.visres.2010.11.005

Malach R, Amir Y, Harel M, Grinvald A (1993) Relationship between intrinsic connections and functional architecture revealed by optical imaging and in vivo targeted biocytin injections in primate striate cortex. Proc Natl Acad Sci USA 90(22):10469-10473

Mannan SK, Pambakian ALM, Kennard C (2010) Compensatory strategies following visual search training in patients with homonymous hemianopia: an eye movement study. J Neurol 257(11):1812-1821. doi:10.1007/s00415-010-5615-3

Mattingley JB, Davis G, Driver J (1997) Preattentive filling-in of visual surfaces in parietal extinction. Science 275(5300):671-674. doi:10.1126/science.275.5300.671

Njomboro P, Deb S, Humphreys GW (2008) Dissociation between decoding and reasoning about mental states in patients with theory of mind reasoning impairments. J Cogn Neurosci 20(9):1557-1564. doi:10.1162/jocn.2008.20118

Nothdurft HC (1992) Feature analysis and the role of similarity in preattentive vision. Percept Psychophys 52(4):355-375. doi:10.3 758/BF03206697

Nothdurft HC, Gallant JL, Van Essen DC (2000) Response profiles to texture border patterns in area V1. Vis Neurosci 17(3):421-436. doi:10.1017/S0952523800173092
Pavlovskaya M, Sagi D, Soroker N, Ring H (1997) Visual extinction and cortical connectivity in human vision. Cogn Brain Res 6(2):159-162. doi:10.1016/S0926-6410(97)00028-1

Pavlovskaya M, Sagi D, Soroker N (2000) Contrast dependence of perceptual grouping in brain-damaged patients with visual extinction. Spat Vis 13(4):403-414. doi:10.1163/156856800741289

Piccini C, Lauro-Grotto R, Del Viva MM, Burr D (2003) Agnosia for global patterns: when the cross-talk between grouping and visual selective attention fails. Cogn Neuropsychol 20(1):3-25. doi:10.1080/02643290244000167

Polat U, Sagi D (1993) Lateral interactions between spatial channels: suppression and facilitation revealed by lateral masking experiments. Vision Res 33(7):993-999. doi:10.1016/0042-6989(93)90081-7

Polat U, Sagi D (1994) Spatial interaction in human vision: from near to far via experience dependent cascades of connections. Proc Natl Acad Sci 91:1206-1209

Ptak R, Valenza N, Schnider A (2002) Expectation-based attentional modulation of visual extinction in spatial neglect. Neuropsychologia 40(13):2199-2205. doi:10.1016/S0028-3932

Riddoch MJ, Humphreys GW, Edwards S, Baker T, Willson K (2003) Seeing the action: neuropsychological evidence for actionbased effects on object selection. Nat Neurosci 6(1):82-89. doi:10.1038/nn984

Riddoch MJ, Humphreys GW, Jacobson S, Pluck G, Bateman A, Edwards M (2004) Impaired orientation discrimination and localisation following parietal damage: on the interplay between dorsal and ventral processes in visual perception. Cogn Neuropsychol 21(6):597-623. doi:10.1080/02643290342000230

Riddoch MJ, Humphreys GW, Hickman M, Clift J, Daly A, Colin J (2006) I can see what you are doing: action familiarity and affordance promote recovery from extinction. Cogn Neuropsychol 23(4):583-605. doi:10.1080/02643290500310962

Riddoch MJ, Chechlacz M, Mevorach C, Mavritsaki E, Allen HA, Humphreys GW (2010a) The neural mechanisms of visual selection: the view from neuropsychology. Annu N Y Acad Sci 1191(1):156-181. doi:10.1111/j.1749-6632.2010.05448.x

Riddoch MJ, Scott SB, Humphreys GW (2010b) No direction home: extinction is affected by implicit motion. Cortex 46(5):678-684. doi:10.1016/j.cortex.2009.05.013

Roelfsema PR, Lamme VAF, Spekreijse H, Bosch H (2002) Figureground segregation in a recurrent network architecture. J Cogn Neurosci 14(4):525-537. doi:10.1162/08989290260045756

Romani A, Callieco R, Tavazzi E, Cosi V (2003) The effects of collinearity and orientation on texture visual evoked potentials. Clin Neurophysiol 114(6):1021-1026. doi:10.1016/ S1388-2457(03)00075-0

Ross WD, Grossberg S, Mingolla E (2000) Visual cortical mechanisms of perceptual grouping: interacting layers, networks, columns, and maps. Neural Netw 13(6):571-588. doi:10.1016/ S0893-6080(00)00040-X

Scholte HS, Jolij J, Fahrenfort JJ, Lamme VAF (2008) Feedforward and recurrent processing in scene segmentation: electroencephalography and functional magnetic resonance imaging. J Cogn Neurosci 20(11):2097-2109. doi:10.1162/jocn.2008.20142

Seghier ML, Vuilleumier P (2006) Functional neuroimaging findings on the human perception of illusory contours. Neurosci Biobehav Rev 30(5):595-612. doi:10.1016/j.neubiorev.2005.11.002

Seghier ML, Friston KJ, Price CJ (2007) Detecting subject-specific activations using fuzzy clustering. NeuroImage 36(3):594-605. doi:10.1016/j.neuroimage.2007.03.021

Seghier ML, Ramlackhansingh A, Crinion J, Leff AP, Price CJ (2008) Lesion identification using unified segmentation-normalisation models and fuzzy clustering. NeuroImage 41(4):1253-1266. doi:10.1016/j.neuroimage.2008.03.028

Snow JC, Miranda RR, Humphreys GW (2013) Impaired visual sensitivity within the ipsilesional hemifield following 
parietal lobe damage. Cortex 49(1):158-171. doi:10.1016/j. cortex.2011.07.005

Ursino M, La Cara GE (2004) A model of contextual interactions and contour detection in primary visual cortex. Neural Netw 17:719735. doi:10.1016/j.neunet.2004.03.007

Vangkilde S, Habekost T (2010) Finding Wally: prism adaptation improves visual search in chronic neglect. Neuropsychologia 48(7):1994-2004. doi:10.1016/j.neuropsychologia.2010.03.020

VanRullen R, Delorme A, Thorpe S (2001) Feed-forward contour integration in primary visual cortex based on asynchronous spike propagation. Neurocomputing 38-40:1003-1009. doi:10.1016/ S0925-2312(01)00445-3

Vivas AB, Humphreys GW, Fuentes LJ (2008) Object-based inhibition of return in patients with posterior parietal damage. Neuropsychology 22(2):169-176. doi:10.1037/0894-4105.22.2.169

Vuilleumier PO, Rafal RD (2000) A systematic study of visual extinction: between- and within-field deficits of attention in hemispatial neglect. Brain 123:1263-1279. doi:10.1093/brain/123.6.1263

Vuilleumier PO, Schwartz S (2001) Modulation of visual perception by eye gaze direction in patients with spatial neglect and extinction. NeuroReport 12(10):2101-2104
Vuilleumier PO, Schwartz S, Verdon V, Maravita A, Hutton C, Husain M, Driver J (2008) Abnormal attentional modulation of retinotopic cortex in parietal patients with spatial neglect. Curr Biol 18(19):1525-1529. doi:10.1016/j.cub.2008.08.072

Wagemans J, Elder JH, Kubovy M, Palmer SE, Peterson MA, Singh M, von der Heydt R (2012) A century of Gestalt psychology in visual perception: I. Perceptual grouping and figure-ground organization. Psychol Bull 138(6):1172-1217. doi:10.1037/ a0029333

Ward R, Goodrich S, Driver J (1994) Grouping reduces visual extinction: neuropsychological evidence for weightlinkage in visual selection. Visual Cogn 1(1):101-129. doi:10.1080/13506289408402295

Watson AB, Pelli DG (1983) QUEST: a Bayesian adaptive psychometric method. Percep Psychophys 33(2):113-120

Wilson JT, Singer W (1981) Simultaneous visual events show a longrange spatial interaction. Percep Psychophys 30(2):107-113. doi: 10.3758/BF03204467

Yen S-C, Finkel LH (1998) Extraction of perceptually salient contours by striate cortical networks. Vision Res 38(5):719-741. doi:10.1016/S0042-6989(97)00197-1 